\title{
GRUPO DE APOIO ÀS PESSOAS COM DOENÇA DE PARKINSON E SEUS FAMILIARES
}

\author{
Angela Maria Alvarez \\ Universidade Federal de Santa Catarina \\ alvarez@ccs.ufsc.br \\ Lucia Isako Takasi Gonçalves \\ Universidade Federal de Santa Catarina \\ lhtakase@gmail.com \\ Jordelina Schier \\ Universidade Federal de Santa Catarina \\ nina.schier@gmail.com
}

\author{
Karina Silvieira de Almeida Hammerschmidt \\ Universidade Federal de Santa Catarina \\ karina.h@ufsc.br \\ Bruna Caroline de Souza \\ Universidade Federal de Santa Catarina \\ karina.h@ufsc.br \\ Rafaela Vivian Valcarenghi \\ Universidade Federal de Santa Catarina \\ rafaelavalcarenghi@yahoo.com.br
}

Erro! Fonte de referência não encontrada.

Relato de experiência com objetivo descrever as vivências do projeto de extensão - Grupo de apoio às pessoas com doença de Parkinson e seus familiares no âmbito da Universidade Federal de Santa Catarina. Esse projeto utiliza a tecnologia cuidativa de ajuda mútua, interdisciplinaridade e abordagem humanística, em que pessoas e membros familiares que partilham de um mesmo problema de vida/sofrimento buscam apoio para seu fortalecimento através da troca de experiências de suas estratégias de enfrentamento. O projeto por meio das atividades desenvolvidas proporciona alívio de estresses e inseguranças, reflete sobre aspectos legais e éticos referentes à doença; estimula a participação em órgãos de controle social. Tecnologias de cuidado adaptado às necessidades específicas da pessoa com doença de Parkinson e seus familiares podem contribuir na produção do conhecimento específico para área da Enfermagem e oferecem subsídios para atenção a pessoa com doença de Parkinson e seus familiares cuidadores.

Palavras-chave: Doença de Parkinson. Tecnologia cuidativa. Promoção da Saúde

\section{SUPPORT GROUP FOR PEOPLE WITH PARKINSON DISEASE AND THEIR FAMILIES}

\begin{abstract}
Experience Report with Objective describe how Extension Project Experiences - Support Group for People with Parkinson's disease and ITS Family no Scope of Federal University of Santa Catarina. This project uses the Mutual Aid Technology cuidativa, interdisciplinary and Humanistic Approach in que People and Family Members What share of hum EVEN Problem of life / Grief Support paragraph seek His strength through the Strategies YOUR Experiences Exchange confrontation. The through the activities developed Project provides relief from stress and insecurities, reflects on Legal and ethical issues related to the disease; It encourages participation in organs of social control. Care technologies Adapted At Specific Needs of People with Parkinson's disease and HIS Family CAN contribute to the Specific Knowledge Production paragraph area of Nursing and offer Subsidies paragraph Care for People with Parkinson's disease and HIS Family caregivers.
\end{abstract}

Keywords: Parkinson disease. Care technology. Health Promotion 


\section{GRUPO DE APOYO PARA PERSONAS CON ENFERMEDAD DE PARKINSON Y SUS FAMILIAS}

\section{Resumen}

Relato de experiencia con el fin de describir las experiencias de los proyectos de extensión - grupo de apoyo para personas con enfermedad de Parkinson y sus familias en la Universidad Federal de Santa Catarina. Este proyecto utiliza la tecnología cuidativa ayuda mutua, enfoque interdisciplinario y humanista, en el que las personas y familiares que comparten el mismo problema de la vida / sufrimiento buscando apoyo para su fortalecimiento a través del intercambio de experiencias de sus estrategias de afrontamiento. El proyecto por medio de las actividades proporciona un alivio del estrés y la inseguridad, que se refleja en los aspectos legales y éticos relacionados con la enfermedad; fomenta la participación en los órganos de control social. Tecnologías de Atención a la medida de las necesidades específicas de la persona con enfermedad de Parkinson y sus familias pueden contribuir en la producción de conocimientos específicos en el campo de la enfermería y proporcionar cuidado adicional para la persona con la enfermedad de Parkinson y sus cuidadores familiares.

Palabras clave: Enfermedad de Parkinson. Tecnología Cuidativa. Promoción de la Salud 


\section{INTRODUÇÃO}

Uma condição crônica de saúde caracteriza-se pelo acometimento de problema de saúde geralmente com instalação lenta, contínua e progressiva, gerando significativo impacto para as pessoas, exigindo tratamento e controle sistemático para prevenir seu agravamento. Segundo Trentini et. al. (2005), a doença crônica pode representar uma ameaça aos projetos de vida dessas pessoas, uma vez que os indivíduos que as possuem são forçados a manter controle rigoroso no seu estilo de vida, caso não controlados, podem levar a situações graves e até à morte. As incapacidades podem ocasionar dependência física, cognitiva e social, representando significativo impacto na sociedade, pois podem resultar em limitações nas atividades de seu viver diário (ELIOPOULOS, 2011).

Os idosos representam um grupo populacional em que as doenças crônicas estão presentes em maior prevalência, sendo que mais de 80\% deles têm pelo menos uma doença crônica (ELIOPOULOS, 2011). Nesse cenário a enfermagem tem papel relevante na promoção do cuidado específico às demandas impostas por essas condições. Esse grupo, em especial, necessita de apoio para o cuidado, que vai além das intervenções tradicionais, pois em sua maioria, enfrentam dificuldades diante de processos de doença somadas às demais alterações do envelhecimento humano (TRENTINI et. al., 2005). Em virtude dessa condição deve-se promover a pessoa idosa, sua independência, autonomia, enfatizando a importância das políticas públicas coerentes com as necessidades de assistência e proporcionando suporte a família (CHAIMOWICZ; CAMARGOS, 2011).

No viver envelhecendo, problemas de saúde ganham maior importância em sua caracterização, entre eles a doença de Parkinson, a qual foi descrita pela primeira vez em 1817, quando James Parkinson publicou sua monografia intitulada " $A$ essay on the shaking palsy - Um ensaio sobre a paralisia agitante", que foi "caracterizada por tremor de repouso, bradiscinesia (lentidão dos movimentos), postura encurvada para frente, marcha festinante". Mais tarde, JeanMartin Charcot acrescentou as características da doença "anormalidades no tônus muscular e na cognição", propondo o nome da doença de Parkinson devido a primeira descrição (PINHEIRO, 2011, p. 285).

Uma das doenças crônicas que merece atenção por parte dos profissionais de saúde e políticas públicas é a doença de Parkinson, pois pode afetar a qualidade de vida das pessoas e suas famílias.

A doença de Parkinson trata-se da segunda doença neurodegenerativa mais frequente das desordens de movimento. Estudos americanos e europeus estimam que anualmente cerca de 8,6 
a 19 pessoas em cada 100 mil habitantes desenvolvam a doença. De acordo com estimativas o número total de pessoas com doença de Parkinson com idade superior a 50 anos nos cinco países mais populosos da Europa ocidental e nos 10 países mais populosos do mundo aumentará de 4,1 a 4,6 milhões (dados de 2005) para 8,7 a 9,3 milhões, no ano de 2030. Nesse sentido, o Brasil será o $5^{\circ}$ país com maior número de portadores da doença, com aproximadamente 340 mil pessoas acometidas (SOUTO, 2009).

Essa patologia refere-se a um transtorno neurológico, de natureza progressiva, caracterizada pela "degeneração das células da camada ventral da parte compacta da substância nigra e do lócus ceruleus". Tratando-se de sua epidemiologia a doença de Parkinson representa 80\% dos casos de parkinsonismo, ocorrendo principalmente em pessoas com mais de 50 anos, e sua incidência aumenta em média 1,5\% em pessoas com mais de 65 anos e 2,5\% em indivíduos com 85 anos e mais, sendo que sua prevalência ocorre em cerca de 550 por 100.000 aos 70 anos de idade. A causa da doença de Parkinson ainda é desconhecida, porém sabe-se que diferentes mecanismos etiopatogênicos podem estar relacionados, como: "fatores genéticos, toxinas ambientais, estresse oxidativo e anormalidade mitocondriais" (PINHEIRO, 2011, p. 285, 286).

É importante salientar que a doença de Parkinson, por tratar-se de uma doença crônica e progressiva e, que devido suas características clínicas, pode levar a incapacidades, isolamento, depressão, dependência, necessidade de cuidado por parte dos familiares, entre outras implicações, e que merece atenção por parte dos profissionais de saúde no sentido de conhecer sua especificidade e adequar os cuidados requeridos. Devido os aspectos mencionados faz-se relevante que os profissionais de saúde, em especial os enfermeiros, tenham conhecimento do processo de envelhecimento, bem como as doenças e limitações que podem acontecer nessa faixa etária, a fim de elaborarem intervenções adequadas para uma melhor qualidade de vida, e também, pensar na assistência de suas famílias, buscando um viver saudável (NAVARROPETERNELLA; MARCON, 2009).

A família diante da progressividade da doença deve ser foco do cuidado, pois compartilha dos mesmos sentimentos e é o suporte social mais importante ao idoso com doença de Parkinson. (NAVARRO-PETERNELLA; MARCON, 2009).

Podemos notar que o Brasil vem preparando-se para atender essa nova demanda da população, através de políticas públicas de saúde, porém há muito que se avançar, é fundamental também, que os profissionais de saúde assumam o compromisso de oferecer a população idosa atenção à saúde que priorize aspectos para a promoção do envelhecimento saudável.

No âmbito da Universidade Federal de Santa Catarina um grupo de professoras e pesquisadoras do Departamento de Enfermagem foi procurado por idosos que possuíam a 
doença de Parkinson solicitando auxílio para criação de um grupo de apoio, uma vez que essas pesquisadoras já possuíam ampla experiência no trabalho junto aos familiares cuidadores de idosos com doença de Alzheimer (ALVAREZ et. al., 2002). Foi assim que as pesquisadoras do Grupo de Estudos sobre Cuidados em Saúde de Pessoas Idosas - GESPI/PEN/UFSC, há dez anos, criaram o projeto de extensão - Grupo de apoio às pessoas com doença de Parkinson e seus familiares no âmbito da UFSC. Tal projeto está vinculado às atividades de extensão do Departamento de Enfermagem da Universidade Federal de Santa Catarina e conta com o apoio do Núcleo de Estudos da Terceira Idade - NETI/UFSC. As atividades relacionadas ao projeto de extensão mobilizaram, em 2004, a criação da Associação Parkinson Santa Catarina (APASC) pelos próprios participantes do grupo de apoio.

Diante das considerações aqui tecidas, o estudo tem como objetivo principal: descrever a experiência do projeto de extensão - Grupo de apoio às pessoas com doença de Parkinson e seus familiares no âmbito da UFSC.

\section{MÉTODOS}

Trata-se de relato de experiência baseado no projeto de extensão - Grupo de apoio às pessoas com doença de Parkinson e seus familiares no âmbito da UFSC. Esse projeto tem como metodologia de trabalho a tecnologia cuidativa de ajuda mútua, interdisciplinaridade e abordagem humanística, em que pessoas e membros familiares que partilham de um mesmo problema de vida/sofrimento e buscam apoio para seu fortalecimento através da troca de experiências.

A sistematização do cuidado em grupo visa propiciar momentos de solidariedade, cooperação, superação e estímulo à pessoa com doença de Parkinson e seus familiares, promovendo assim, apoio para aqueles que, muitas vezes, sentem receio do modo como a doença de Parkinson pode interferir e comprometer a sua vida diária. A ajuda mútua numa perspectiva humanística permite ao enfermeiro, numa posição de horizontalidade ao aproximar-se da realidade da pessoa que vivencia as dificuldades da condição crônica e incapacitante da doença.

Essa tecnologia de cuidado é desenvolvida seguindo as estratégias: Grupo de Ajuda Mútua, que ocorre nas primeiras e terceiras terças-feiras de cada mês às $15 \mathrm{~h} 00 \mathrm{~min}$ horas no auditório do Núcleo de Estudos da Terceira Idade (NETI), que acolhe as atividades do Grupo de Apoio, do qual participam pessoas com doença de Parkinson, familiares e cuidadores, bem como equipe formada por professores, profissionais, acadêmicos e voluntários; Serviço de Apoio e Orientação às Pessoas com Doença de Parkinson e seus Familiares, ocorre por meio de atendimento telefônico, por 
Grupo de apoio às pessoas com Doença de Parkinson e seus familiares

internet e pelo correio com apoio de bolsistas; Visitas domiciliares, que são realizadas por voluntários e pessoas acometidas pela doença que se dispõem a visitar os portadores restritos aos seus domićlios por agravamento de sua condição de saúde; Busca ativa, realizada pela equipe de enfermagem por meio de contato sistematizado com as pessoas com a doença de Parkinson cadastradas e que, por algum motivo se ausentaram das atividades oferecidas; Reuniões comemorativas, que ocorrem em datas especiais com o intuito de promover a integração e socialização das pessoas com a doença, familiares cuidadores e profissionais que atuam junto ao grupo; Atividades Integrativas, organizadas pelos participantes do grupo, exemplo disso é o jogo de bocha, através do qual busca estimular a criatividade, a manter-se socialmente integrado, melhorar a psicomotricidade além de favorecer o condicionamento físico; Atividades educativoinformativas, tem como objetivo informar a comunidade em geral sobre a doença de Parkinson, diagnóstico, tratamento e como melhor lidar com a doença, através da distribuição de cartilha, flayres, palestras e contato pelo telefone e site da Associação Parkinson Santa Catarina.

O projeto de extensão desde o seu início preocupou-se em articular com outras áreas profissionais para oferecer uma proposta interdisciplinar de trabalho. Nesse sentido, atividades relacionadas à atividade física, artes plásticas, psicologia, fisioterapia, medicina e fonoaudiologia foram articuladas para uma atenção integral à pessoa com doença de Parkinson. Com o decorrer do tempo, novas parcerias foram estabelecidas a ponto de constituir um "Programa de Atenção à pessoa com doença de Parkinson e seus familiares", cujas atividades complementares ao grupo de apoio são: Fonoandiologia, atividade realizada todas as quartas-feiras com a participação de profissionais e acadêmicos da fonoaudiologia; Fisioterapia, atividade realizada as terças, quintas e sextas-feiras, com o apoio da Universidade do Estado de Santa Catarina (UDESC) e Universidade Estácio de Sá; Musculação e dança, atividades desenvolvidas por profissionais da educação física, que visam à melhoria das condições de equilíbrio, musculatura e condicionamento físico.

No decorrer desse projeto, surgiu a necessidade de se organizarem para fazerem-se representar. Foi fundada então, a Associação Parkinson de Santa Catarina, que como entidade jurídica, sem fins lucrativos surge como parceira essencial para as atividades propostas pelo Grupo de Apoio.

Integrar o ensino, a extensão e a pesquisa sempre foi uma das diretrizes do trabalho desenvolvido pelo projeto, garantindo, dessa forma, espaço para realização do processo pedagógico baseados na visão integral do ser humano e norteados pela ajuda mútua, além de contribuir para a formação pessoal e profissional de acadêmicos das áreas de saúde e social. 


\section{RESULTADOS E ANÁLISE}

O impacto da doença de Parkinson afeta todos os membros da família, pois além dos aspectos financeiros, toda organização familiar, psicológica e social sofre alterações. Tal fato representa desafio aos enfermeiros e gestores das políticas sociais e de saúde, pois as pessoas com doença de Parkinson e seus familiares carecem de atenção e cuidados por um longo período de tempo.

A parceria existente entre o GESPI e o NETI da Universidade Federal de Santa Catarina com a APASC, visa estabelecer maior contato das pessoas com a doença de Parkinson e seus familiares contribuindo na ampliação do conhecimento acerca da doença e formas de cuidar do doente, realizando atividades para a promoção da saúde e prevenção dos agravos da pessoa com Parkinson, orientando o familiar cuidador, discutindo questões legais de modo que os participantes possam estar inseridos de maneira objetiva e atuante nos órgãos responsáveis pela legislação referente aos direitos da pessoa com a doença de Parkinson.

O projeto de extensão tem tido muitos êxitos como: o atendimento a 180 pessoas com doença de Parkinson; o apoio para as pessoas que descobrem a doença e seus familiares cuidadores; a criação da APASC e o fortalecimento desta; o empoderamento das pessoas com a doença e seus familiares cuidadores; a inserção social; a participação em atividades comunitárias e recreativas; o combate ao preconceito ao idoso e à pessoa idosa em relação à própria doença; a divulgação da doença para o conhecimento da comunidade em geral; a melhoria da qualidade de vida das pessoas com doença de Parkinson e seus familiares cuidadores; a informação sobre aspectos legais e éticos referentes à doença; a participação em órgãos de controle social. Essas atividades favorecem a integração entre as pessoas, melhora da autoestima do paciente com doença de Parkinson, e cultiva o sentimento de pertencimento a um grupo.

Como reforça estudo de Gonçalves et. al. (2010), realizado nesse contexto, as políticas públicas de atenção ao idoso devem enfatizar a promoção da saúde, manutenção da autonomia e valorização de redes de suporte social, gerando impactos nas diversas formas de se prestar assistência aos idosos e em especial aos idosos portadores da doença de Parkinson.

A convivência em grupo é uma forma de se sentir útil e de pertencer como parte de um coletivo. A prática tem demonstrado, pelas muitas experiências positivas de grupos de ajuda mútua, apoio psicossocial às pessoas as quais passam por eventos traumáticos e estressantes, que esse tipo de organização grupal é recurso valioso e pouco oneroso na preservação da saúde mental da coletividade que o vivencia (GONÇALVEZ; ALVAREZ; ARRUDA, 2007). 
Outro estudo realizado nesse contexto mostra a importância de promover as pessoas com doença de Parkinson, por diversas maneiras, dependendo das necessidades de cada indivíduo. Uma das formas de promover um viver saudável refere-se às tecnologias cuidativas, baseadas em ajuda mútua através de grupo voltado as pessoas com doença de Parkinson e seus familiares. Tal tecnologia contribui na formulação de instrumentos que facilitam a reinserção social do idoso com a doença. Soma-se ainda a contribuição do aspecto de promoção da cidadania, à medida que no grupo as pessoas são atendidas com respeito, privacidade e sem discriminação por sua condição de cronicidade e de envelhecimento, e têm a garantia de tomar decisões quanto à própria vida, além de sentirem-se mais fortalecidas para reivindicar seus direitos em setores públicos e privados e na sociedade em geral (SENA et. al., 2010).

Segundo as autoras, ao longo da implantação do grupo de ajuda mútua para portadores da doença de Parkinson e familiares, observaram-se alguns benefícios, como: a aprendizagem entre pares, o aumento da rede de relações, enfrentamento das limitações impostas pela doença, reconhecimento e valorização das potencialidades, facilitação ao acesso aos serviços sociais e de saúde, bem como o exercício da cidadania como ser de direitos (SENA et. al., 2010).

É necessário empreender esforços para que as pessoas com doença de Parkinson e suas famílias possam viver melhor com a doença. Isto pode ser alcançado com uma maior proximidade dos profissionais, de modo que o atendimento prestado, incluindo orientações e informações sobre a doença, sua evolução e formas de enfrentamento, correspondem às necessidades reais dos pacientes e suas famílias (NAVARRO-PETERNELLA; MARCON, 2012).

\section{CONSIDERAÇÕES FINAIS}

O programa de extensão ora referido corrobora intensamente com a assistência que deve ser fornecida às pessoas com doença de Parkinson e seus familiares cuidadores, de forma a garantir o bem estar biopsicossocial dessas pessoas.

Observa-se que o cuidado em grupo estimula as pessoas a buscarem conhecimento sobre seu problema de saúde, compartilha vivências sobre sua doença e favorece o sentimento de pertencimento, importante para a autoestima e a autoconfiança, de forma a diminuir o isolamento, favorecendo a inserção social e a convivência com a condição de cronicidade.

$\mathrm{O}$ aumento no número de pessoas com doença de Parkinson e familiares que foram beneficiados pelo projeto evidencia a relevância de propostas como esta, que estimula a organização da sociedade civil e é de suma importância para a população que envelhece e vê na 
Grupo de apoio às pessoas com Doença de Parkinson e seus familiares

assistência as pessoas idosas com doenças crônico-degenerativas um importante desafio a ser superado a cada ano. E, certamente, o projeto de extensão que foi aqui relatado pode ser encarado com modelo de assistência baseada na articulação da extensão, ensino e pesquisa no âmbito universitário.

Não há dúvida que hoje existe grande preocupação com as pessoas em condição crônica de saúde. As pesquisas cada vez mais enfatizam a utilidade dos estudos estarem buscando desenvolver estratégias de cuidado físico, mental e social, tendo como objetivo a valorização da capacidade funcional dessas pessoas, bem como a manutenção da autonomia e a independência, porém percebe-se que ainda existem poucos estudos relacionados à doença de Parkinson no país. Nesse sentido, o presente relato de experiência visa contribuir para os profissionais de saúde voltar suas atividades de assistência, pesquisa e extensão às pessoas que convivem com esse tipo de doença.

Espera-se com esse projeto consolidar o desenvolvimento de tecnologias de cuidado adaptado às necessidades específicas da pessoa com doença de Parkinson e seus familiares; produção de conhecimento específico para área da Enfermagem; fortalecer as entidades organizativas das pessoas com doença de Parkinson e oferecer subsídios para atenção com pessoas idosas com Parkinson e seus familiares cuidadores.

\section{REFERÊNCIAS}

ALVAREZ, A. M. et. al. Grupo de Ajuda Mútua de Familiares de Idosos Portadores de Doença de Alzheimer e Doenças Similares do HU/UFSC. Revista Ciências da Saúde, v. 21, p. 54-66, 2002.

CHAIMOWICZ, F. CAMARGOS, M. C. S. Envelhecimento e saúde no Brasil. IN: FREITAS, E. V. de.; PY, L. et. al. Tratado de Geriatria e Gerontologia. $3^{a}$ ed. Rio de Janeiro: Guanabara Koogan, 2011, p. 74-98.

ELIOPOULOS, C. Enfermagem gerontológica. $7^{\mathrm{a}}$ ed. Porto Alegre: Artmed, 2011.

GONÇALVES, L. H. T. et. al. Vivencia corporal para portadores de doenca de parkinson: efeitos na capacidade funcional. Geriatria \& Gerontologia, v. 4, p. 62-68, 2010.

GONÇALVES, L. H. T.; ALVAREZ, A. M.; ARRUDA, M. C. Pacientes portadores da doença de Parkinson: significado de suas vivências. Acta Paulista de Enfermagem, v. 20, n. 1, p. 62-8, 2007. 
Grupo de apoio às pessoas com Doença de Parkinson e seus familiares

NAVARRO-PETERNELLA, F. M.; MARCON, S. S. Descobrindo a doença de Parkinson: impacto para o parkinsoniano e seu familiar. Revista Brasileira de Enfermagem, Brasília, fev, v. 62, n. 1, p. 25-31, 2009.

NAVARRO-PETERNELLA, F. M.; MARCON, S. S. Quality of life of a person with Parkinson's disease and the relationship between the time of evolution and the severity of the disease. Revista Latino-Americana Enfermagem, Ribeirão Preto, v. 20, n. 2, apr. 2012.

PINHEIRO, J. E. S. Doença de Parkinson e outros transtornos do movimento. IN: FREITAS, E. V. de.; PY, L. et. al. Tratado de Geriatria e Gerontologia. $3^{\mathrm{a}}$ ed. Rio de Janeiro: Guanabara Koogan, 2011, p. 285-291.

SENA, E. L .S. et. al. Tecnologia cuidativa de ajuda mútua grupal para pessoas com Parkinson e suas famílias. Texto Contexto Enfermagem, Florianópolis, jan-mar, v. 19, n. 1, p. 93-103, 2010.

SOUTO, M. O. Era outono em Barcelona: o meu encontro com Mr. Parkinson. Editora Pathernon: Niterói, Rio de Janeiro, 2009.

TRENTINI, M. et. al. Enfrentamento de situações adversas e favoráveis por pessoas idosas em condições crônicas de saúde. Revista Latino-Americana de Enfermagem, São Paulo, v. 13, n. 1, p. 3845, jan./ fev. 2005. 